\title{
On Stapedectomy in Old Fenestration Cavities
}

\author{
L.B.W. \\ Jongkees
}

From the E.N.T. Department (Head: Prof. Dr. L. B. W. Jongkees) of the Wilhelmina Gasthuis, Amsterdam

Author's address: Prof. L. B.W. Jongkees, M.D., Koninginnenweg 157, Amsterdam-Z. (Netherlands)

Description of 8 patients operated upon for bad hearing after fenestration for otosclerosis.

The technique according to Schuknecht is used with a little piece of fat tissue connected to the manubrium of the malleus by a hook of stainless steel wire. In order to keep the wire prothesis on the extremely mobile manubrium mallei well fixed, an extra small hook is attached to it. In all patients we had a primary good improvement.

In 6 patients a closure of the airbone gap was achieved. In one case cochlear function decreased after some weeks ending in a moderate postoperative loss of function.

In one patient with practically complete deafness the result of the operation gave him back sufficient hearing to use a hearing aid with success. 